\title{
Totem et tabou dans le conflit du Kosovo : remarques sur les limites naturelles d'une médiation internationale
}

\author{
Victor-Yves Ghebali Hebali
}

\author{
(2) OpenEdition \\ Journals \\ Édition électronique \\ URL : http://journals.openedition.org/conflits/314 \\ DOI : $10.4000 /$ conflits.314 \\ ISSN : 1777-5345 \\ Éditeur : \\ CCLS - Centre d'études sur les conflits lilberté et sécurité, L'Harmattan \\ Édition imprimée \\ Date de publication : 1 mars 2000 \\ ISBN : 2-7384-9385-8 \\ ISSN : $1157-996 \mathrm{X}$ \\ Référence électronique \\ Victor-Yves Ghebali Hebali, «Totem et tabou dans le conflit du Kosovo : remarques sur les limites \\ naturelles d'une médiation internationale », Cultures \& Conflits [En ligne], 37 | printemps 2000, mis en \\ ligne le 20 mars 2006, consulté le 30 mars 2021. URL : http://journals.openedition.org/conflits/314 ; \\ DOI : https://doi.org/10.4000/conflits.314
}

Ce document a été généré automatiquement le 30 mars 2021.

Creative Commons License 


\title{
Totem et tabou dans le conflit du Kosovo : remarques sur les limites naturelles d'une médiation internationale
}

\author{
Victor-Yves Ghebali Hebali
}

1 La guerre dont le Kosovo a été le théâtre du 24 mars au 10 juin 1999 a suscité un large débat qui, d'emblée, s'est polarisé sur les points nodaux de la légalité juridique et de la légitimité politique de l'intervention militaire de l'OTAN. Les critiques de celle-ci ont généralement soutenu que le recours à la force avait été déclenché de manière inconsidérée, alors que l'option diplomatique demeurait encore ouverte ${ }^{1}$. Au-delà de sa charge passionnelle, l'argument mérite attention en ce qu'il affirme qu'un compromis politique restait envisageable. Poser le problème en ces termes linéaires est sans doute acceptable dans le cadre d'une polémique où il ne saurait y avoir de "vérités », mais des positions subjectives également défendables - qu'il s'agisse des motivations « humanitaires » de l'intervention militaire ou des visées « hégémoniques » de l'OTAN. En fait, du point de vue de Sirius, la vraie question n'est pas de savoir si l'option diplomatique fut abandonnée trop vite, de manière délibérée ou non, en 1999. Elle est de comprendre plutôt pourquoi toutes les tentatives de règlement pacifique, entreprises dès 1992-1993 et reprises de manière intensive à partir de 1998, ne furent qu'une tâche de Sisyphe. On soutiendra ici que l'affaire du Kosovo appartient à une catégorie bien particulière de conflits: celle dont le règlement est normalement concevable soit à l'issue d'une ordalie agonistique consacrant de manière décisive la victoire de l'un des protagonistes sur le plan militaire, soit sous l'effet d'une intervention coercitive extérieure armée ou non armée - de l'ONU, d'une organisation régionale mandatée par celle-ci ou, encore, d'une coalition ad hoc d'Etats agissant avec (ou sans) le blanc seing de l'organisation mondiale. Le conflit du Kosovo était réfractaire aux procédures diplomatiques de règlement pacifique pour au moins trois raisons majeures : la nature non négociable de ses enjeux, la mentalité solipsiste de la partie serbe et, enfin, les failles de l'approche "totémique» des médiateurs 
internationaux. La nature non négociable des enjeux du conflit Né dans le sillage de la guerre balkanique de 1912-1913, lorsque Serbes et Monténégrins reprirent à l'Empire ottoman un territoire peuplé par une majorité albanaise, le problème du Kosovo demeura pratiquement en dehors des relations internationales jusqu'en 1989, date de l'abolition par Slobodan Milosevic du statut de province autonome dont bénéficiait le territoire en question dans la République de Tito. Dans ses données brutes, le problème se ramenait à la coexistence inégalitaire de deux peuples européens (séparés par la langue et la religion) sur un même territoire vis-à-vis duquel chacun d'eux estimait avoir des titres prioritaires. Le terme « inégalitaire » n'est pas déplacé : l'oppression et l'exploitation systématiques pratiquées au Kosovo par le groupe numériquement minoritaire, mais politiquement dominant (les Serbes), à l'encontre de la majorité albanaise dès la reconquête du territoire introduisit au sein de celui-ci des rapports de type colonial ${ }^{2}$. En tout cas, ce qui à l'époque de Tito pouvait apparaitre comme une question de protection des droits individuels et collectifs d'une minorité nationale compacte (c'est-à-dire majoritaire au plan local) au sein d'une fédération multiethnique redevint, du fait de l'acte de Slobodan Milosevic, une question coloniale. Le problème du Kosovo est généralement lu à travers la grille des " conflits ethniques ». Abstraction faite des difficultés sémantiques et conceptuelles soulevées par la notion fourre-tout d'« ethnie ", une telle lecture n'est acceptable qu'à condition d'attribuer ici à l'ethnicité le rôle d'une variable emblématique et instrumentale - mais nullement causale ${ }^{3}$. A ne s'en tenir qu'aux apparences, le conflit du Kosovo oppose bien deux peuples séparés par certains marqueurs identitaires clés de l'ethnicité : la langue et la religion. Serbes et Albanais parlent des langues qui, en dépit de leur commune appartenance génétique à la famille indo-européenne, ne sont pas compréhensibles pour leurs locuteurs respectifs ${ }^{4}$. En même temps, les Serbes sont orthodoxes alors que les Albanais pratiquent majoritairement l'Islam. Le marqueur linguistique est prégnant pour les Albanais qui sont l'unique peuple des Balkans dont l'identité nationale s'est formée exclusivement sur la base de la langue - et chez qui, vu l'existence de minorités orthodoxes et catholiques, la religion n'est pas un facteur décisif de cohésion ${ }^{5}$. En revanche, pour les Serbes, la frontière orthodoxie/islam apparaît fondamentale pour deux raisons précises. D'une part, du fait de la participation active de l'Eglise orthodoxe aux mouvements d'émancipation politique et aux guerres de libération des deux derniers siècles, l'orthodoxie constitue une composante identitaire majeure des peuples chrétiens des Balkans ${ }^{6}$. D'autre part, l'aversion des Serbes à l'égard des Albanais ne s'explique pas seulement par un antagonisme religieux classique, aggravé par le grief (imaginaire) du « fondamentalisme »; elle repose avant tout sur une tare que les Serbes attribuent aux Albanais : être les descendants de " renégats »- de chrétiens convertis à l'islam à partir du XVIème siècle par pur intérêt économique et depuis lors totalement acquis à la cause de l'ennemi héréditaire turc ${ }^{7}$. On pourrait donc poser que l'altérité des Albanais est perçue dans une optique politico-culturelle à connotation essentiellement religieuse, alors que celle des Serbes est vécue sur un mode avant tout politique où le culturel n'intervient que comme un facteur aggravant: les Serbes représentent l'oppresseur politique qui de surcroît parle une autre langue et pratique une religion différente. Mais quels que soient leur réalité et leur poids, ces marqueurs identitaires ne pouvaient ipso facto constituer une source directe ou profonde de conflit. Au Kosovo, ils servirent de "lentille grossissante", au travers de laquelle Albanais et Serbes interprétèrent leur coexistence imposée ainsi que leur lien ancestral avec un territoire censé leur appartenir en vertu de titres de propriété fondés sur une série de 
considérations d'ordre historique, juridique et même (pour ce qui est des Serbes) mystique. Sur le plan historique, le débat tourne autour de l'identification du " premier occupant " du territoire. Les Albanais font ainsi valoir leur qualité de descendants directs des plus anciens habitants des Balkans (les Illyriens) qui s'installèrent à l'ouest de la péninsule balkanique aux alentours du VIIème siècle avant $\mathrm{J}$.-C., soit très longtemps avant les tribus slaves dont l'apparition dans la région n'est attestée qu'à partir de la fin du VIème siècle après J.-C.; en outre, cette occupation aurait été continue, contrairement au cas des Serbes qui quittèrent le Kosovo en masse au XVIIème siècle pour n'y revenir qu'au début du XXème siècle après la reconquête du territoire ${ }^{8}$. Pour leur part, les Serbes soutiennent avoir pris possession du territoire avant les Albanais dont les tribus, longtemps confinées dans les montagnes, ne seraient descendues dans les plaines du Kosovo qu'après la conquête turque et le grand exode serbe de 1690 vers l'empire austro-hongrois. Alimentée par des arguments historiquement peu ou guère vérifiables, cette disputatio paraît bien vaine. Au niveau $\mathrm{du}$ droit international, la controverse se déroule sur un terrain en apparence plus ferme: depuis l'éclatement de la Yougoslavie de Tito, les Albanais du Kosovo se réclament du principe du droit des peuples à disposer d'eux-mêmes et les Serbes du principe de l'intégrité territoriale des Etats. Les premiers se réfèrent à la pratique bien établie des Nations Unies selon laquelle tout peuple vivant sous domination coloniale ou d'autres formes de domination (occupation étrangère et discrimination raciale du type apartheid) peut légitimement exercer son droit à l'autodétermination. Or, les seconds refusent d'admettre l'existence d'une situation coloniale au Kosovo, tout en faisant valoir que le droit international positif ne reconnaît un droit à la sécession ni aux minorités nationales ni aux peuples. A cela, les Albanais rétorquent qu'un tel droit peut se justifier dans des circonstances exceptionnelles telles que l'oppression systématique, massive et continue d'une minorité nationale par l'Etat qui l'abrite. Mais le débat ne s'arrête pas là. Les Serbes le prolongent sur le terrain de l'irrationnel en couronnant leur argumentaire par l'affirmation du caractère «sacré » du territoire du Kosovo. Celui-ci, soutiennent-ils, représente un «lieu saint » en tant que berceau de l'Eglise orthodoxe serbe, aire d'expansion du plus prestigieux des royaumes de la Serbie médiévale et lieu de la bataille du Kosovo Polje (1389) - bataille perdue contre les Turcs, mais néanmoins considérée comme le symbole de l'héroïsme, de la prise de conscience nationale ainsi que du début du martyre multiséculaire du peuple serbe. Outre que la province englobe bien une plaine riche en vestiges religieux serbes, cette vision ne correspond guère à la réalité. Le royaume serbe en question formait un amalgame multiethnique de Serbes, de Grecs, d'Albanais et d'autres peuples de la région. Quant à la bataille du Kosovo, elle fut davantage un combat entre princes qu'un choc civilisationnel : elle opposa les Turcs à une coalition de sept chefs balkaniques, dont deux Albanais alors... chrétiens ${ }^{9}$ ! En fait, le lien « sacré » que la nation serbe entretient avec le Kosovo relève avant tout du surnaturel, comme l'atteste cette déclaration significative d'un membre de l'Eglise orthodoxe serbe en 1983: «Le Kosovo n'est pas seulement un lieu de séjour, mais aussi une création métaphysique... Le ciel aussi bien que la terre constitue cette patrie serbe. Le noumène de l'esprit dans le phénomène du temps et de l'espace. C'est la meilleure preuve que pour définir l'appartenance à un sol, ce n'est pas seulement la composition numérique, dominante de l'habitat qui est l'essentiel, mais aussi, et peut-être plus, cette création spirituelle que le peuple a engendrée et dans laquelle il existe sur un mode existentiel supérieur. L'idéogenèse est dans ce cas plus importante que l'ethnogenèse ${ }^{10}$. Sur la base de ce lien mystique, les 
Serbes revendiquent ce qu'une essayiste française a appelé leurs "droits de l'âme ", revendication sacrée face à laquelle celle - profane - des droits de l'homme, individuels ou collectifs des Albanais du Kosovo ne saurait guère peser ${ }^{11}$. C'est au niveau de ce débat que l'ethnicité révèle sa vraie fonction : celle de valeur-refuge justifiant, pour les uns, une lutte existentielle contre l'oppression et, pour les autres, une lutte vécue comme non moins existentielle pour le contrôle du destin du territoire. Dès lors, la difficulté que pose le problème du Kosovo du point de vue de la médiation internationale apparaît dans sa plénitude. Pour les Albanais, la situation de domination coloniale imposée par un peuple slave à un peuple non slave ne peut avoir qu'une issue logique : l'indépendance - et non plus l'autonomie, option valable pendant un temps au sein de la Yougoslavie multiethnique de Tito mais devenue caduque depuis le coup de force de 1989. Celui-ci, en démontrant que les Serbes ne concevaient les relations que dans la domination et la répression, a par là même détruit la confiance qui est à la base de toute forme de coexistence volontaire acceptable. De toute façon, l'éclatement de la Yougoslavie n'a-t-il pas révélé que la coexistence des Serbes avec d'autres peuples (fussent-ils slaves) était devenue impossible? Dans ces conditions, il n'existe aucune raison valable de refuser aux Albanais du Kosovo le droit à l'autodétermination exercé par un grand nombre de peuples européens depuis l'effondrement du communisme ${ }^{12}$. Mais pour les Serbes, une telle perspective représente un scandale métaphysique autant que politique. La même remarque vaut aussi pour toute formule d'autonomie visant à restaurer le statut particulier - aboli en 1989 - qui faisait du Kosovo une entité fédérale disposant de la maîtrise pratiquement totale de ses affaires internes, sans passer par l'intermédiaire de la Serbie. Ce double rejet imposait à la politique serbe une unique voie: celle de la mise au pas totale de la majorité albanaise. Refus de l'indépendance et refus de toute solution autre que l'indépendance : tels apparaissaient les enjeux - mutuellement exclusifs - de la question du Kosovo à la veille de l'intervention militaire de l'OTAN. Des enjeux d'une telle nature ne peuvent faire l'objet d'un véritable marchandage politique. Le statut d'un territoire à valeur mystique pour les Serbes ou les modalités d'une subjugation coloniale, avec ou sans garanties internationales pour les Albanais ne sont pas des questions négociables. Est-ce à dire que nulle solution pacifique n'était concevable? Il est toujours permis de croire qu'un conflit entre des groupes d'êtres rationnels comporte certaines "clés " à partir desquelles un compromis pacifique pourrait être aménagé. Toutefois, le problème dépend de la capacité des protagonistes à percevoir l'existence de telles « clés » et, plus encore à en faire usage sur la base du principe de réalité ainsi qu'avec une certaine dose d'empathie. Les Albanais se devaient ainsi de prendre en compte le fait que l'option de l'indépendance était inacceptable pour la communauté internationale comme pour les Serbes. Le principe de réalité leur commandait donc d'accepter un statut d'autonomie aussi substantiel que possible pour une période intérimaire de durée raisonnable. Tel fut d'ailleurs la position qu'ils adoptèrent - pour de simples raisons tactiques - à l'issue du processus de Rambouillet. Du côté serbe, les données se présentaient autrement: comment conserver le plein contrôle d'un territoire "sacré " peuplé d'une majorité écrasante d'Albanais désireux de se séparer de la mini-Yougoslavie? Le principe de réalité dictait à cet égard une réponse claire : donner aux Albanais des raisons valables de continuer à lier leur sort à Belgrade. Mais Milosevic, tributaire d'un type bien particulier de mentalité, s'avéra incapable d'aborder le problème dans une optique autre que solipsiste. La «mentalité solipsiste» de la partie serbe ${ }^{13}$. ${ }^{14}$ La position traditionnelle serbe sur la question du Kosovo illustre à souhait le cas de figure où il 
n'existe pour le sujet d'autre réalité que lui-même (solus ipse) au sein d'un système de valeurs clos forgé pour ses besoins exclusifs. Convaincus de la justesse de leur cause, les ethnonationalistes serbes ont en effet érigé celle-ci en absolu face auquel toute autre considération - émanant soit des Albanais, soit de la communauté internationale devenait irrecevable. Dans la mesure où la vénération pour le territoire sacré du Kosovo fait ontologiquement abstraction des Albanais, il y a bel et bien solipsisme. Pour les ethnonationalistes serbes, le problème du Kosovo ne se ramène pas au respect des droits démocratiques de la majorité albanaise, mais au drame d'un peuple subissant l'humiliation de vivre comme une simple minorité dans le berceau (réel ou perçu comme tel) de sa nation ethnique. Ce fut bien dans cette optique que, dès la reconquête du Kosovo, les ethnonationalistes serbes entreprirent de re-slaviser celui-ci par des mesures discriminatoires d'ordre économique (réforme agraire) et culturelles que le Royaume de Yougoslavie appliqua sur une plus grande échelle dans l'entre-deuxguerres. Après 1945, Tito eut le mérite de rompre avec le solipsisme. Confronté aux contraintes de la realpolitik, il estima opportun d'accorder au Kosovo un statut d'autonomie qui, au terme de réajustements successifs, finit par devenir réel et substantie ${ }^{15}$. En conséquence, la revendication constante formulée par les Albanais à partir de 1968 se limita - significativement - à la transformation de la province en république fédérée. En d'autres mots, sous Tito, la coexistence imposée aux Albanais offrit malgré tout à ceux-ci assez d'avantages pour ne pas les inciter à réclamer la fusion avec l'Albanie ou même l'indépendance. Elle les poussa seulement à revendiquer l'égalité politique et juridique avec les peuples slaves de la Fédération. Mais, avec Slobodan Milosevic, les Serbes revinrent de manière quasi jubilatoire au solipsisme. Il suffit de se référer ici trois à faits significatifs: l'abolition inconstitutionnelle de l'autonomie du Kosovo, la répression systématique et brutale de la longue résistance pacifique des Albanais et, enfin, le refus de tout compromis à Rambouillet sur la question de l'autonomie de la province. Milosevic abolit l'autonomie du Kosovo pour le compte de la Serbie en 1989 sans l'accord, constitutionnellement indispensable, de toutes les autres entités de la Fédération et, en tout cas, comme s'il n'existait pas de majorité albanaise sur le territoire. Réalisé sous la contrainte de la force armée, cet acte illégal se déroula par ailleurs dans un climat de triomphalisme provocateur, comme s'il s'agissait d'une juste punition: les «droits de l'âme " prenaient leur revanche sur les droits de l'homme... La réaction immédiate des Albanais au coup de force serbe fut mesurée et pacifique puisqu'elle consista à proclamer le Kosovo "République fédérée " de la Yougoslavie (1990). Les autorités serbes ne surent pas saisir ce qui constituait peut-être encore l'une des dernières possibilités de règlement politique. Au contraire, elles y rétorquèrent par l'instauration de l'état d'urgence, la suppression de tous les pouvoirs locaux résiduels et la mise en place d'un impitoyable dispositif répressif. A toutes ces mesures, les Albanais répondirent encore par une désobéissance civile non violente, avant de proclamer plus tard l'indépendance de la République du Kosovo à l'issue d'un référendum clandestin (1991) et de mettre sur pied des structures parallèles d'administration sous la présidence d'Ibrahim Rugova. La politique solipsiste de Belgrade finit par radicaliser les Albanais au point de favoriser l'émergence, en 1996, d'une "Armée de libération du Kosovo » (UCK) bien décidée à obtenir l'indépendance du Kosovo par les armes. Le solipsisme atteignit son apogée à l'occasion du processus de Rambouillet. Rappelons que celui-ci ne visait pas à détacher le Kosovo de la Yougoslavie, mais à doter la province d'un statut d'autonomie substantiel dans le cadre de l'intégrité territoriale de la fédération serbo-monténégrine. Or, à Rambouillet, la 
position serbe sur la question de l'autonomie se ramenait à un double rejet. D'une part, les Serbes récusaient toute formule visant à faire du Kosovo, en fait ou en droit, une entité fédérale : compte tenu de sa nature «sacrée ", le territoire devait demeurer sous la juridiction directe (entendons : le joug) de la Serbie. D'autre part, ils se refusaient à tirer les conséquences du fait majoritaire albanais, c'est-à-dire à reconnaître aux Albanais des droits ou avantages liés à leur situation majoritaire. Affirmant que le Kosovo englobait une demi-douzaine de "communautés nationales » non-albanaises totalisant quelque 650000 personnes (250 000 Serbes/Monténégrins, 150000 Musulmans, 150000 Roms ainsi que plus de 100000 Turcs, Croates, Egyptiens, Goranis Serbes musulmans de la région de Gora - «Egyptiens » et autres), Belgrade estimait en conséquence que l'autonomie ne devait être accordée que sur la base de l'égalité de toutes ces communautés quelle qu'en soit l'importance numérique respective et, de surcroît, avec droit de veto individuel ${ }^{16}$. Autrement dit, l'idée était d'introduire au Kosovo des structures locales strictement égalitaires qui, sous le couvert d'un fédéralisme en trompe-l'œil, auraient permis à la minorité serbe de s'opposer (seule ou avec l'appui de n'importe quelle autre minorité non-albanaise) aux vœux de la majorité albanaise. L'étiologie de ce refoulement persistant du réel peut être appréhendée sous deux angles. D'une part, elle se rattache à l'existence d'un vieux "complexe victimaire ». Comme l'a montré Catherine Lutard, certaines contraintes géographiques ont favorisé chez les Serbes non seulement une mentalité collective sensible aux menaces d'encerclement physique et de conspiration internationale, mais aussi l'autoimage d'un "peuple martyr " ${ }^{17}$. Ce fut à partir de ce sentiment diffus, partagé et entretenu par l'Eglise orthodoxe, que les ethnonationalistes serbes récusèrent, à la veille de l'éclatement de la Yougoslavie, la possibilité de vivre en tant que communautés minoritaires au milieu d'autres peuples. En fait, il convient de reconnaître que le système politique serbe ne pouvait alors gérer les changements en cours (dont la chute du communisme et le relâchement des liens fédéraux), d'autant plus que sa culture politique ne lui offrait pas « les catégories qui [rendaient] lisible ou supportable le changement » et qu'une partie de la population "se [sentait] menacée » par les changements en question ${ }^{18}$. Révélateur d'un certain dysfonctionnement de la relation du soi à l'autrui, une telle auto-image incitait à faire percevoir toute différence, réelle ou imaginaire, comme irréversiblement perverse, tout en permettant au sujet de se poser lui-même en justicier-bourreau ${ }^{19}$. D'autre part, le refus serbe de la réalité doit être interprété à la lumière du moment historique précis au cours duquel le Kosovo fut privé de son autonomie. Le geste de Milosevic reflétait sans doute la volonté d'un apparatchik communiste de fonder son pouvoir naissant sur le mythe légitimant du Kosovo qui, depuis la mort de Tito avait recommencé à obséder la conscience collective serbe - en particulier avec le « Memorandum » soumis à l'Académie serbe des sciences et des arts, en septembre 1986, par un groupe d'intellectuels anonymes ${ }^{20}$. Il n'en constituait pas moins, consciemment ou non, un acte de "haine identitaire" compensatoire à la perte des repères identitaires ressentie par les Serbes parallèlement à la chute du communisme et au développement de processus centrifuges au sein de la Yougoslavie multiethnique. On sait que la haine identitaire a notamment pour fonction " d'ancrer l'identité vacillante du sujet sur un môle artificiel qui résulte de la projection de toutes les traces de son altérité intérieure, à l'origine de la fêlure de son support identitaire, sur un Autre affecté abusivement d'une altérité tout aussi artificielle ${ }^{21}$. Elle pousse également le sujet - dont l'identité est par définition toujours multipolaire à s'enliser dans une option mono-identitaire introvertie ${ }^{22}$. Dans le cas d'espèce, elle 
pourrait expliquer pourquoi Slobodan Milosevic, pourtant décrit par tous ceux qui l'ont approché comme un acteur froidement réaliste, ait pu faire totale abstraction de cette maxime politique rousseauiste d'élémentaire bon sens : « le plus fort n'est jamais assez fort pour être toujours le maître, s'il ne transforme sa force en droit, et l'obéissance en devoir " ${ }^{23}$. En somme, liée au départ à un complexe victimaire ancien, la crise du Kosovo a éclaté dans une Yougoslavie en proie à une crise identitaire qui était en même temps une crise de légitimité de l'Etat ${ }^{24}$. Slobodan Milosevic fit tout le reste en "[portant] à l'incandescence la singularité serbe dans ce qu'elle pouvait avoir de sombre ${ }^{25}$. Les failles de l'approche «totémique » de la médiation internationale Bien que l'anthropologie moderne semble avoir désormais renoncé à considérer la notion de totémisme (qui englobe le couple totem/tabou) comme «la clef qui ouvrirait presque toutes les serrures » ${ }^{26}$, il n'est pas incongru de la transposer mutatis mutandis aux relations internationales et plus particulièrement à la gestion médiatrice du conflit du Kosovo. Dans cette optique, on constatera que les acteurs du système international (toutes catégories confondues, mais en particulier les acteurs-Etats) se réclament d'un même totem emblématique: les "Nations Unies", expression pouvant désigner l'Organisation des Nations Unies, la Charte des Nations Unies ou, encore, la doctrine issue de la pratique des grands organes des Nations Unies en matière de droits de l'homme, de décolonisation, de développement, etc. En tout cas, le texte canonique qu'est la Charte des Nations Unies édicte clairement un certain nombre d'interdits à valeur de tabou - à commencer par celui fondamental entre tous du non-recours à la menace ou à l'emploi de la force contre l'intégrité territoriale ou l'indépendance politique des Etats. Bien que souvent transgressés, et servant ainsi symboliquement de "repas totémique ", les divers tabous de la Charte n'en relèvent pas moins de ce que Roger Caillois appelait un "impératif catégorique négatif " d'ordre supérieur ${ }^{27}$. Dans l'affaire du Kosovo, les instances internationales chargées de tâches médiatrices se sont heurtées à deux tabous spécifiques : celui de l'intervention dans les affaires intérieures des Etats et celui de la violation de l'intégrité territoriale des Etats. Le tabou de l'intervention ne fut convenablement géré, c'est-à-dire surmonté, qu'au terme d'une longue période de tâtonnements récurrents. Il convient de rappeler à cet égard que la communauté internationale ne réagit pas à l'abolition de l'autonomie du Kosovo au motif qu'il s'agissait d'un problème de type purement interne. Elle ne commença à se préoccuper de l'affaire qu'à partir de l'automne 1992, soit après l'éclatement de la Yougoslavie, et avec l'accord du pouvoir fédéral yougoslave alors détenu par le prooccidental Milan Panic. Dans le cadre de la Conférence internationale de Genève sur l'ex-Yougoslavie, l'ONU entreprit ainsi d'ébaucher un certain dialogue entre Belgrade et la direction des Albanais du Kosovo. De son côté, l'OSCE s'attacha, à travers les bons offices d'une poignée de diplomates regroupés au sein d'une "Mission de longue durée ", à apaiser la situation des droits de l'homme sur le terrain. Dans les deux cas, l'objectif visé était de désamorcer ce qui apparaissait désormais comme une bombe à retardement. Cette double expérience de diplomatie préventive tourna cependant court après les élections de décembre 1992 : en remplaçant Milan Panic aux commandes de la Fédération, Slobodan Milosevic décréta que le Kosovo était une affaire interne ne nécessitant nulle médiation internationale. La communauté internationale s'inclina devant le tabou invoqué et cessa de se manifester pratiquement jusqu'en mars 1998, c'est-à-dire jusqu'à l'entrée en scène de l'UCK. La transformation du Kosovo en un théâtre d'attentats armés et de répression sanglante à l'encontre de la population civile albanaise introduisait une donnée nouvelle : un risque sérieux d'escalade régionale sur 
fond de catastrophe humanitaire. Vu ce risque, l'OSCE estima que la crise du Kosovo ne pouvait plus être considérée comme une affaire purement intérieure. En conséquence, elle soumit à Belgrade un "plan d'action » politique que Slobodan Milosevic rejeta aussitôt comme concernant des questions relevant exclusivement de la souveraineté yougoslave. Peu après, en avril 1998, Milosevic fit massivement avaliser par référendum le refus de toute médiation étrangère au Kosovo. Cette nouvelle victoire du tabou de la non-intervention fut cependant la dernière. En octobre 1998, sous la menace crédible de frappes militaires de l'OTAN ainsi que d'une intense pression directe de la diplomatie américaine, Belgrade finit par admettre, dans une déclaration unilatérale, que la question du Kosovo constituait bel et bien une affaire d'intérêt international. Cette reconnaissance ne demeura pas de pure forme : elle fut assortie de l'acceptation de l'établissement au Kosovo de deux "missions de vérification" - l'une terrestre (OSCE) et l'autre aérienne (OTAN) ${ }^{28}$. La gestion du tabou de l'intégrité territoriale s'avéra, en revanche, plus difficile. En effet, le Groupe de contact, l'instance qui s'arrogea la gestion politique du conflit sans mandat formel des Nations Unies, se prononça constamment en faveur du respect de l'intégrité territoriale de la Yougoslavie et, par voie de conséquence, contre l'indépendance du Kosovo. Sa démarche fondamentale, qui consista à exhorter les autorités de Belgrade et les Albanais du Kosovo à ouvrir sans conditions un dialogue direct en vue d'un règlement excluant à la fois l'option de l'indépendance et celle d'un simple réaménagement du statu quo, emprunta les formes successives des bons offices, de la médiation et de la conciliation dirigée. Dans un premier stade, le Groupe de contact se contenta de poser que la solution à rechercher devrait permettre de doter la province d'un "statut amélioré » comportant "un degré significatif d'autonomie réelle » et sauvegardant les droits civiques de tous les habitants albanais ou non. Vu le blocage de la situation, il estima par la suite que le dialogue devait s'engager sous son égide directe, sans tête à tête, sur la base de propositions de travail concrètes formulées par ses soins ${ }^{29}$. Finalement, en janvier 1999, devant la persistance de l'impasse, il imposa aux deux parties de se rendre à Rambouillet en vue de négocier un règlement définitif au cours d'une période maximum de 21 jours. En offrant comme solution de fond un nouveau statut d'autonomie pour le Kosovo - idée jugée totalement dépassée par la majorité des Albanais et considérée comme inacceptable par la partie serbe - le Groupe de contact se plaçait lui-même dans l'impasse. Dans ces conditions, l'échec du processus de règlement mené par le Groupe de contact était prévisible. Les arrangements proposés à Rambouillet exigeaient des deux protagonistes du conflit des sacrifices inacceptables. Pour les Albanais, il s'agissait de renoncer à la lutte armée et, qui plus est, à l'indépendance. Pour les Serbes, il s'agissait d'accepter un statut d'autonomie qui tout en respectant le principe de l'intégrité territoriale de la Yougoslavie, aurait fait de la province une entité territoriale d'un genre particulier échappant, comme sous Tito à l'emprise de la Serbie ${ }^{30}$. En outre, un tel statut n'aurait été valable que pour une période de trois ans au terme de laquelle "une réunion internationale sera convoquée en vue de définir un mécanisme pour un règlement définitif pour le Kosovo, sur la base de la volonté du peuple, de l'avis des autorités compétentes, des efforts accomplis par chacune des Parties dans la mise en œuvre du présent Accord ... ", clause que les Serbes interprétaient comme une promesse larvée d'indépendance ${ }^{31}$. Circonstance aggravante, la surveillance du respect des arrangements de Rambouillet incomberait non seulement à une «Mission de mise en œuvre » OSCE/Union européenne (chargée de nombreuses fonctions civiles dont la police et la sécurité publique), mais aussi à une force établie 
par l'OTAN sur mandat de l'ONU et dotée de larges pouvoirs en matière militaire - soit à un dispositif perçu par Belgrade comme destiné à instituer un protectorat de facto sur le Kosovo. Le texte de l'accord proposé par le Groupe de contact fut rejeté par les Serbes qui conclurent aussitôt, avec les délégués de six "communautés nationales " non-albanaises ainsi qu'avec deux petits partis politiques albanais collaborationnistes et non représentatifs, un accord spécial attribuant au «Kosovo-Metohija » une autonomie de façade ${ }^{32}$. En revanche, les Albanais acceptèrent le texte de Rambouillet, pour des raisons purement tactiques: assurés de la défection des Serbes, ils y apposèrent leur signature aux fins de démontrer de quel côté se situait l'absence de volonté de compromis ${ }^{33}$. De tous les facteurs explicatifs des limites et de l'impasse finale de la médiation internationale dans le conflit du Kosovo, le plus déterminant aura sans doute été l'enlisement des ethnonationalistes serbes dans un solipsisme autodestructeur. Fondé sur une conception introvertie et mystique de l'ethnicité, ce solipsisme (que l'on ne saurait évidemment imputer à l'ensemble du peuple serbe) excluait par définition une communication positive avec les Albanais du Kosovo. Par là même, il ne pouvait que convaincre ceux-ci de l'impossibilité de la coexistence et de l'inanité de la non violence. De fait, il les poussa à franchir le pas de la lutte armée et de l'indépendantisme. Dans un tel cas de figure, la question de l'épuisement des moyens de règlement pacifique a peu de sens: en théorie, d'autres formes de médiation demeuraient concevables; mais celles-ci n'auraient pu en l'occurrence aboutir étant donné la détermination des Serbes à rejeter n'importe quelle solution d'autonomie non conforme à leurs vœux. D'un point de vue plus général, le succès d'une entreprise de médiation est surtout tributaire de la bonne volonté et de la bonne foi des parties concernées - paramètres qui, dans l'affaire du Kosovo, faisaient manifestement défaut. Cela dit, on doit reconnaitre que les efforts de médiation internationale furent tardifs (automne 1992), discontinus (du fait de leur interruption de 1993 à 1997) et conceptuellement erronés (vu la soumission de formules de règlement inacceptables pour les deux parties). En tout cas, l'usage de la force armée contre Belgrade prit la relève non seulement d'une année de démarches diplomatiques intensives, mais aussi de dix ans de répression massive à laquelle les Albanais avaient jusqu'en 1997 répondu par la non-violence. L'intervention militaire de l'OTAN était-elle pour autant, politiquement ou moralement, justifiée ? La réponse à cette question constitue une pure affaire d'opinion qui n'a pas à être abordée ici ${ }^{34}$. Il paraît plus judicieux de s'interroger sur les effets de cette intervention. Clairement, celle-ci n'a pas réglé le problème du Kosovo. En favorisant l'instauration d'un protectorat international revêtu du sceau totémique des Nations Unies, elle a certes permis de rompre l'impasse qui prévalait jusqu'alors. En même temps, elle a entraîné une certaine perversion des données humanitaires du problème, puisque c'est le sort des communautés ethniques non albanaises qui fait maintenant l'objet de préoccupation. Mais, surtout, l'intervention n'a guère contribué - du moins jusqu'à ce jour (fin 1999) - à faire évoluer la position de la communauté internationale vis-à-vis du tabou fétichiste de l'intégrité territoriale. En effet, le dispositif institué au Kosovo par la résolution 1244 (adoptée le 10 juin 1999 par le Conseil de sécurité à la fin des opérations de l'OTAN), exclut l'indépendance. Son objectif se limite volontairement, dans l'esprit du texte de Rambouillet, à doter la province d'une autonomie substantielle au sein de la République fédérale de Yougoslavie (Serbie/Monténégro). Or, il convient réalistement d'admettre que l'autonomie constitue une option aujourd'hui tout à fait dépassée. L'indépendance apparait comme une issue logique au motif que le problème du Kosovo a fini par 
devenir un problème de type colonial ${ }^{35}$. Mais il y a plus. N'oublions pas que le régime de Belgrade tira prétexte des bombardements de l'OTAN pour réaliser (à travers le plan dit «Fer à cheval» conçu avant l'intervention militaire) le grand fantasme ethnonationaliste pensé dès 1937 par l'historien serbe Vasa Cubrilovic : la déportation massive des Albanais du Kosovo ${ }^{36}$. Faute de pouvoir repeupler le Kosovo par des Serbes, Slobodan Milosevic avait décidé de dépeupler la province de ses Albanais. Cet acte a durablement, pour ne pas dire irrémédiablement, compromis les chances d'une éventuelle nouvelle coexistence au sein d'un Etat commun. Comme l'a rappelé à juste titre Joseph Krulic, la singularité serbe réside ici dans le fait que de tous les régimes de l'Europe post-communiste, seul celui de Belgrade a cru qu'une telle opération pouvait être à la fois légitime et réalisable ${ }^{37}$. Le tabou de l'intégrité territoriale des Etats est, certes, indispensable au fonctionnement d'un système international tant soit peu stable et ordonné ; en autoriser la "consommation totémique » d'une manière générale mènerait au chaos. Mais aussi fondamental soit-il, le principe de l'intégrité territoriale cesse d'être éthiquement respectable lorsqu'un Etat exerce à l'encontre de populations placées sous sa juridiction une politique inhumaine. La justification d'une telle affirmation découle de l'idée qu'une véritable communauté de nations ne peut être basée que sur des valeurs supérieures à celles du strict respect des frontières des Etats existants. Considérer comme "sacré » un certain territoire peut être compréhensible et même acceptable; mais cela ne saurait justifier de nulle manière un comportement massivement et systématiquement attentatoire aux droits de l'homme. La morale de l'affaire du Kosovo est, en fin de compte, assez claire : une cause «juste » perd toute légitimité si elle est défendue par des méthodes injustes et irrespectueuses de l'humain.

\section{NOTES}

1. Exemplaire à cet égard est l'article d'Olivier Corten, « Tous les moyens diplomatiques avaient-ils réellement été épuisés ? L'échec du plan de Rambouillet », La guerre du Kosovo. Eclairages et commentaires sous la direction de Bernard Adam, Bruxelles, GRIP/Ed. Complexe, 1999 ( Les Livres du GRIP », 230-240), pp. 32-42.

2. Ces rapports sont finement analysés par Michel Roux dans Les Albanais en Yougoslavie. Minorité nationale, territoire et développement, Paris, Editions de la Maison des sciences de l'homme, 1992, pp. 191 et ss.

3. Sur les difficultés de la notion d'« ethnie ", voir les divers articles traitant du thème «Ethnic Nationalism and the World Systemic Crisis ", publiés dans le vol. 19, n 3, 1998 de l'International Political Science Review (en particulier, Fred W. Riggs, « Glossary of Terms Used in this Issue ", pp. 311-330) ainsi que, dans la livraison spéciale de la Revue du M.A.U.S.S. (nº 13, 1er semestre 1999 : Le retour de l'ethnocentrisme. Purification ethnique versus universalisme cannibale), les articles d'Annamaria Rivera, «EthnieEthnicité », pp. 43-60 et de Guy Nicolas, «L'identité et ses mythes », pp. 96-120. Voir aussi notre article « Le semblable et le différent : réflexions sur l'ethnonationalisme dans l'Europe post-communiste », Mélanges en l'honneur de Nicolas Valticos. Droit et justice, Paris, Pedone, 1999, pp. 159-176. 
4. Cf. Bernard Sergent, Les Indo-Européens. Histoire, langues, mythes, Paris, Payot («Bibliothèque Payot »), 1996, pp. 92-93 et Shaban Demiraj, « L'Albanais », Langues indo-européennes sous la direction de Françoise Bader, Paris, CNRS (« Sciences du langage »), 1997, pp. 223-234.

5. Michel Roux, op. cit., p. 205.

6. Radmila Radic, «L'Eglise et la question serbe », Radiographie d'un nationalisme. Les racines serbes du conflit yougoslave (sous la direction de Nebojsa Popov), Paris, Editions de l'Atelier, 1998, pp. 137-138.

7. Christine von Kohl and Wolfgang Libal, « Kosovo. The Gordian Knot of the Balkans », Kosovo in the Heart of the Powder Keg. Compiled and Edited by Robert Elsie, Boulder, East European Monographs ( $\mathrm{N}^{\circ}$ cdlxxviii), 1997, pp. 14-18.

8. Sur les Illyriens, cf. Pierre Cabanes, Les Illyriens de Bardylis à Genthios (IVè - IIè siècles avant J.-C.), Paris, SEDES, 1988 (« Regards sur l'histoire »). Sur l'origine des Slaves, cf. Michel Kazanski, Les Slaves. Les origines, Ier - VIIe siècle après J.C, Paris, Editions Errance, 1999, (« Hespérides »).

9. L'armée ottomane comptait elle aussi d'importants contingents chrétiens. Pour une critique serrée des mythes serbes, cf. Noel Malcolm, Kosovo. A Short History, London, Macmillan, 1998, pp. 22-40 et 62-64.

10. Ce discours de l'archiprêtre Bozidar Mihac est reproduit dans Radic (op. cit.), p. 141. 11. Anne Yelen, Kossovo [sic], 1389-1989. Bataille pour les droits de l'âme, Lausanne, L'âge d'homme, 1989.

12. Tout au long de la guerre froide, l'URSS prétendit que le principe de l'autodétermination n'était pas applicable en Europe, au motif qu'il n'y existait nulle situation de type colonial. Cette thèse (que les Occidentaux parvinrent à contester partiellement dans le cadre du processus de Helsinki) vola en éclat avec l'effondrement du communisme, dans le sillage duquel de nombreux Etats européens exercèrent leur droit à l'autodétermination - sans référence formelle, il est vrai, au colonialisme. Pour plus de détails, cf. notre article : « Le droit des peuples à disposer d'eux-mêmes - de la détente à l'après-communisme ", Arès, vol. XIII/2, 1992, pp. 91-98.

13. Il convient de bien préciser que l'expression " partie serbe » vise les ethnonationalistes de Serbie (ou selon le cas les autorités de Belgrade) et non pas le peuple serbe dans son ensemble.

14. Au sein de la Yougoslavie de Tito, les rapports de type colonial ne disparurent pas pour autant. Ils furent seulement compensés par les modalités d'un régime d'autonomie croissante culminant avec les dispositions de la Constitution fédérale de 1974.

15. Au sein de la Yougoslavie de Tito, les rapports de type colonial ne disparurent pas pour autant. Ils furent seulement compensés par les modalités d'un régime d'autonomie croissante culminant avec les dispositions de la Constitution fédérale de 1974.

16. OSCE, document CIO.GAL/88/98 du 7 décembre 1998.

17. Catherine Lutard, Géopolitique de la Serbie/Monténégro, Bruxelles, Editions Complexe, 1998, pp.13 \& 37.

18. Joseph Krulic, « Réflexions sur la singularité serbe », Le Débat, n 107, novembredécembre 1999, p. 111.

19. Sur ce point, cf. Guy Jucquois, De l'égocentrisme à l'ethnocentrisme ou les illusions de la bonne conscience linguistique, Louvain, Peeters, 1987, (« Bibliothèque des Cahiers de l'Institut de linguistique de Louvain », 31), pp. 5-6. 
20. Dans sa seconde partie, le Memorandum dénonçait le " génocide » en cours au Kosovo contre le peuple serbe et adjurait ce dernier de reconquérir le berceau de sa nation (texte dans : Le nettoyage ethnique. Documents historiques sur une idéologie serbe rassemblés, traduits et commentés par Mirko Grmek, Marc Gjidara et Neven Simac, Paris, Fayard, 1993, pp. 236-269).

21. Guy Nicolas, op. cit., p. 105. Sur le problème du délitement des repères identitaires, cf. François Thual, Les conflits identitaires. Paris, Ellipses, 1995.

22. Guy Nicolas, op. cit., p. 108. Cf. aussi Amin Maalouf, Les identités meurtrières, Paris, Grasset, 1998.

23. Jean-Jacques Rousseau, Du contrat social (phrase introductive du chapitre III du livre premier).

24. On peut considérer que les crises identitaires procèdent normalement d'une crise de légitimité de l'Etat. Cf. Philippe Delmas, Le bel avenir de la guerre, Paris, Gallimard, 1997 («Folio/Actuel », 56), p. 166.

25. Krulic, op. cit., p. 117. Krulic va jusqu'à soutenir que cette singularité qui a « ensauvagé » ou « décivilisé » les Serbes est essentiellement attribuable au fait que ceux-ci n'ont, à aucun moment de leur histoire passée ou présente, accepté comme légitime la domination ottomane. Ce refus d'acculturation « les a empêchés de s'intégrer dans le cadre ottoman, alors même que cette domination destructurait les fondements traditionnels de l'identité serbe "; dans ces conditions, " seule une Eglise orthodoxe repliée sur elle-même et la tradition orale des poèmes épiques célébrant une grandeur passée mythifiée [pouvaient assurer] une fragile identité blessée » (p. 99) fragilité expliquant une «mentalité obsidionale » alimentée par les « mythes historiques », le " fétichisme des différences minuscules » et la constante réactivation d'une sémiologie de type identitaire (p. 100).

26. La formule est de Camille Tarot, De Durkheim à Mauss, l'invention du symbolique. Sociologie et sciences des religions, Paris, Editions La Découverte (« Recherches, Bibliothèque du M.A.U.S.S. »), 1999, p. 511. Depuis l'ouvrage majeur publié en 1962 par Claude Levi-Strauss sur la question (Le totémisme aujourd'hui), le totémisme est généralement considéré comme un système à finalité avant tout classificatoire. 27. Roger Caillois, L'homme et le sacré, édition augmentée, Paris, Gallimard, 1997 (« Folio/Essais », 84), p. 29.

28. Pour plus de détails sur les arrangements du 13 octobre 1998 dits « Accord Holbrooke/Milosevic », cf. notre analyse dans Relations internationales et stratégiques, $\mathrm{n}^{\circ} 33$, printemps 1999.

29. Cette tâche fut dévolue à l'Ambassadeur américain Christopher Hill au cours de 1998.

30. Le point de vue selon lequel le Kosovo aurait ainsi bénéficié de " plus de pouvoirs » que la Serbie ou le Monténégro au sein d'une Yougoslavie transformée « véritablement [en] une confédération » (Corten, op.cit., pp. 35 -37) nous paraît relever d'un jugement de valeur sans fondement sérieux.

31. En posant le principe d'un « règlement définitif ", cette disposition (article 1er $\S 3$ du chapitre 8 de la dernière mouture du texte de Rambouillet) ne faisait pourtant que réserver l'avenir de manière vague. Si elle impliquait que l'autonomie ne devait pas être considérée comme une fin en soi, elle ne retenait pas pour autant l'option de l'indépendance contre laquelle toutes les instances internationales appropriées s'étaient clairement prononcées. 
32. C'est-à-dire une autonomie conçue sur la base de l'égalité absolue des « communautés nationales » du Kosovo et fondamentalement applicable dans le cadre de la République de Serbie. Texte de l'accord : doc. ONU S/1999/302 du 21 mars 1999. 33. Il existe au moins trois versions (anglaises) du projet de Rambouillet respectivement datées des 27 janvier, 2 février et 23 février 1999. Traduction française du dernier projet (daté erronément du 27 mai 1999) sur http:// www.France.diplomatie.fr/actual/dossiers/kossovo76.html

34. Cf. notre article « Le Kosovo entre la guerre et la paix », Défense nationale, 55e année, août-septembre 1999, pp. 62-79.

35. En fait, l'idée d'un partage du Kosovo semble aujourd'hui gagner du terrain au sein de la communauté internationale.

36. Le texte du mémoire de Cubrilovic («L'expulsion des Albanais ») se trouve dans : Le nettoyage ethnique, op. cit., pp. 161-185.

37. Krulic, op. cit., p. 113.

\section{INDEX}

Index géographique : Balkans, Kosovo

Mots-clés : diplomatie, gestion de conflits, gestion de crise, politique étrangère

Index chronologique : 1999 